\title{
Far-Red Carbon Dots as Efficient Light-Harvesting Agent for Enhanced Photosynthesis
}

Dongna Li, Wei Li, Haoran Zhang, Xuejie Zhang, Jianle Zhuang, Yingliang Liu, Chaofan Hu, and Bingfu Lei*

Key Laboratory for Biobased Materials and Energy of Ministry of Education, College of Materials and

Energy, South China Agricultural University, Guangzhou 510642, P. R. China

*Email: tleibf@scau.edu.cn. 


\section{EXPERIMENTAL}

Cell Counting Kit-8 (CCK-8) Assay for the Cell Cytotoxicity: $100 \mu \mathrm{L}$ Hela cell suspension was seeded in a 96-well plate and then incubated at $37{ }^{\circ} \mathrm{C}, 5 \% \mathrm{CO} 2$ for $24 \mathrm{~h}$. The cells were exposed to the another $100 \mu \mathrm{L}$ culture medium that contained different concentrations of FR-CDs $(0,3,5,7$, and 9 $\mu \mathrm{g} / \mathrm{mL}$ ) for $24 \mathrm{~h}$ after the removal of original culture medium. Next, discarded the culture medium and washed the cells 3 times with Dulbecco's phosphate buffered saline (DPBS). $100 \mu \mathrm{L}$ culture medium with $10 \mathrm{ml} \mathrm{CKK-8} \mathrm{into} \mathrm{was} \mathrm{added} \mathrm{into} \mathrm{each} \mathrm{well,} \mathrm{followed} \mathrm{by} \mathrm{incubation} \mathrm{for} 2 \mathrm{~h}$. Finally, the absorbance values of each well were measured by microplate reader at the wavelength of $450 \mathrm{~nm}$.

Hill Reaction: This reaction was conducted to investigate the photosynthetic activity. Under the light irradiation, the electrons that transfer from photosystem II (PS II) to photosystem I (PS I) can be obtained by Electron acceptor. In this work, we used 2, 6-dichlorophenolindophenol (DCPIP) which has a strong affinity for electron as reductant. A suspension of chloroplast ( $5 \mathrm{~mL}$ ), DCPIP and different concentration of FR-CDs $(0,3,6,9,12,15 \mu \mathrm{g} / \mathrm{mL})$ were mixed. The mixtures were irradiated under the xenon lamp at an intensity of $4 \mathrm{~mW} \mathrm{~cm}^{-2}$ for $5 \mathrm{~min}$. Then recorded the absorbance of the mixtures per minute. The change in absorbance of DCPIP represented the reduction of DCPIP. When the variation of CDPIP is higher, the electron transfer rate between the PS II and PS I is faster, and this means that the photosynthetic activity increases. The measurements were carried out in six replicates for each group.

Adenosine Triphosphate (ATP) Production: During the Hill reaction process, the electron transfer process is accompanied by the production of oxygen. The formula of Hill reaction is expressed as 
follows:

$$
2 \mathrm{DCPIP}+2 \mathrm{H}_{2} \mathrm{O} \underset{\text { Chloroplasts }}{\stackrel{\text { Light }}{\underset{\text { }}{\longrightarrow}}} 2 \mathrm{DCPDP}-\mathrm{H}+\mathrm{O}_{2}
$$

We built a simple device to collect oxygen which was released by the Hill reaction. The measurements were carried out six times for each group.

Measurement of ATP Production: To the measurement of ATP production, the equal chloroplasts and different concentration of FR-CDs $(0,3,6,9,12$ and $15 \mu \mathrm{g} / \mathrm{mL})$ were mixed. Then put the mixture under the irradiation of xenon lamp (intensity: $8 \mathrm{~mW} \cdot \mathrm{cm}-2$ ) for $5 \mathrm{~min}$. Next, pour the reaction solution into boiling water and then put them into a boiling water bath for $3 \mathrm{~min}$. The extract was rapidly cooled in an ice bath and subsequently centrifuged at $0{ }^{\circ} \mathrm{C}$ to remove the precipitation. Finally, the ATP production of obtained solution is measured using an ATP Bioluminescent Assay Kit (Beyotime Biotechnology). The measurements were carried out six times for each group.

Calculation of the Absolute Photoluminescence Quantum Yield (QY): The quantum yield was measured using time-resolved spectrofluorometer (Edinburgh, FLS1000). The absolute photoluminescence QY can be calculated by using the following equation:

$$
\mathrm{QY}=\frac{\int I_{\text {emission }}}{\int I_{\text {blank }}-\int I_{\text {sample }}}
$$

Iblank is the spectrum of the light used for excitation with only the solvent; Isample is the spectrum of the light used to excite the CDs; Iemission was the photoluminescence emission spectrum of the CDs sample (all these spectra are collected using the integrating sphere). 


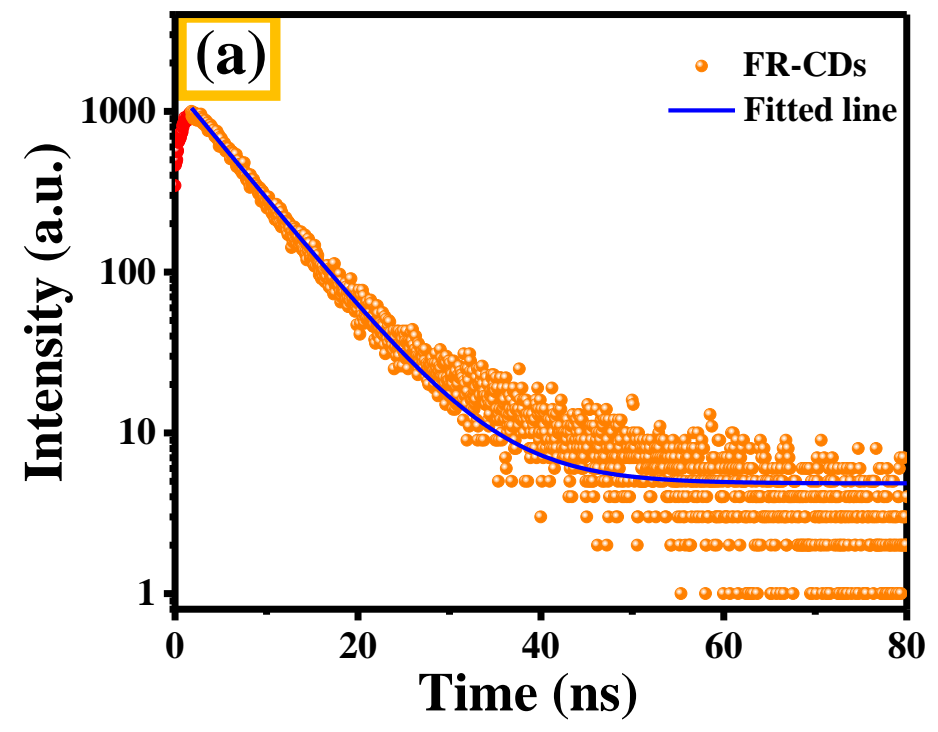

Figure S1. a) Decay curve of FR-CDs monitored at $683 \mathrm{~nm}$.
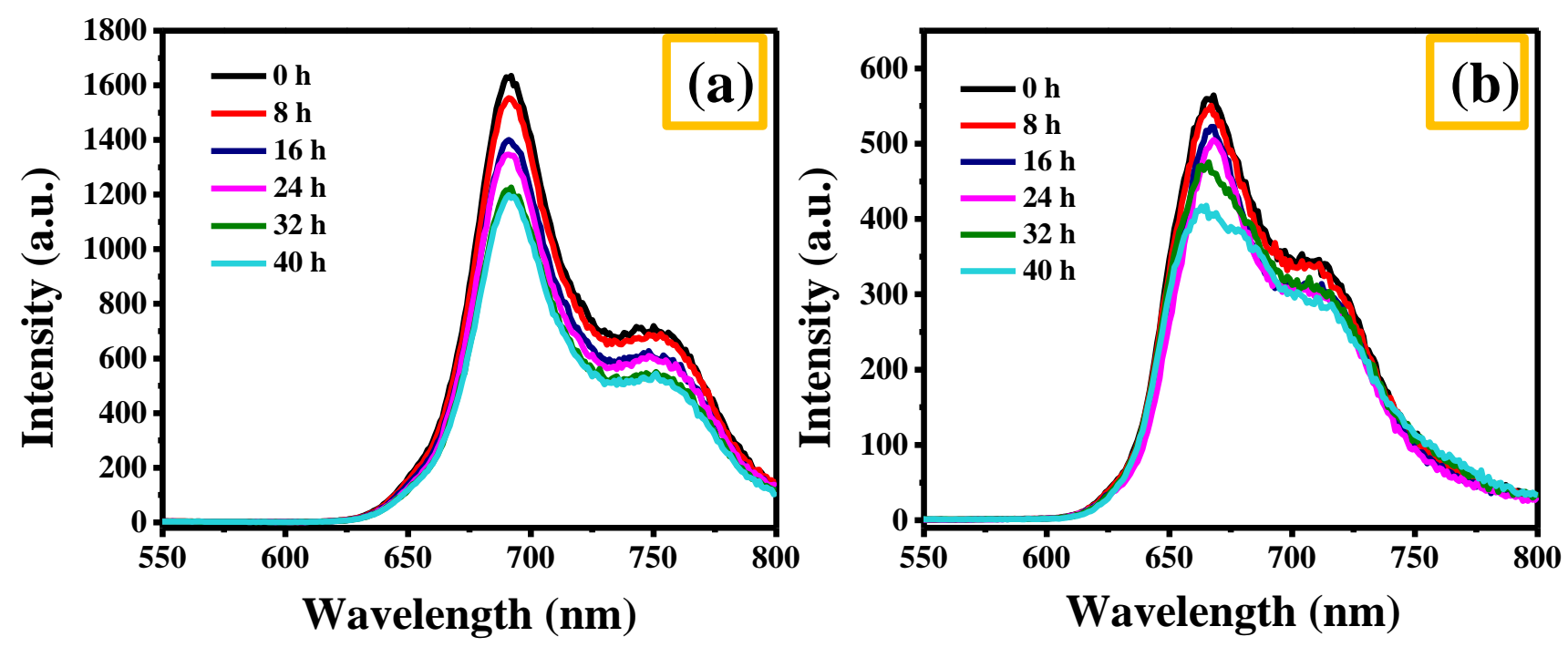

Figure S2. The changes of fluorescence intensity of FR-CDs in aqueous solution (a) and dulbecco's modified eagle medium (DMEM) (b)under the irradiation of UV light. 

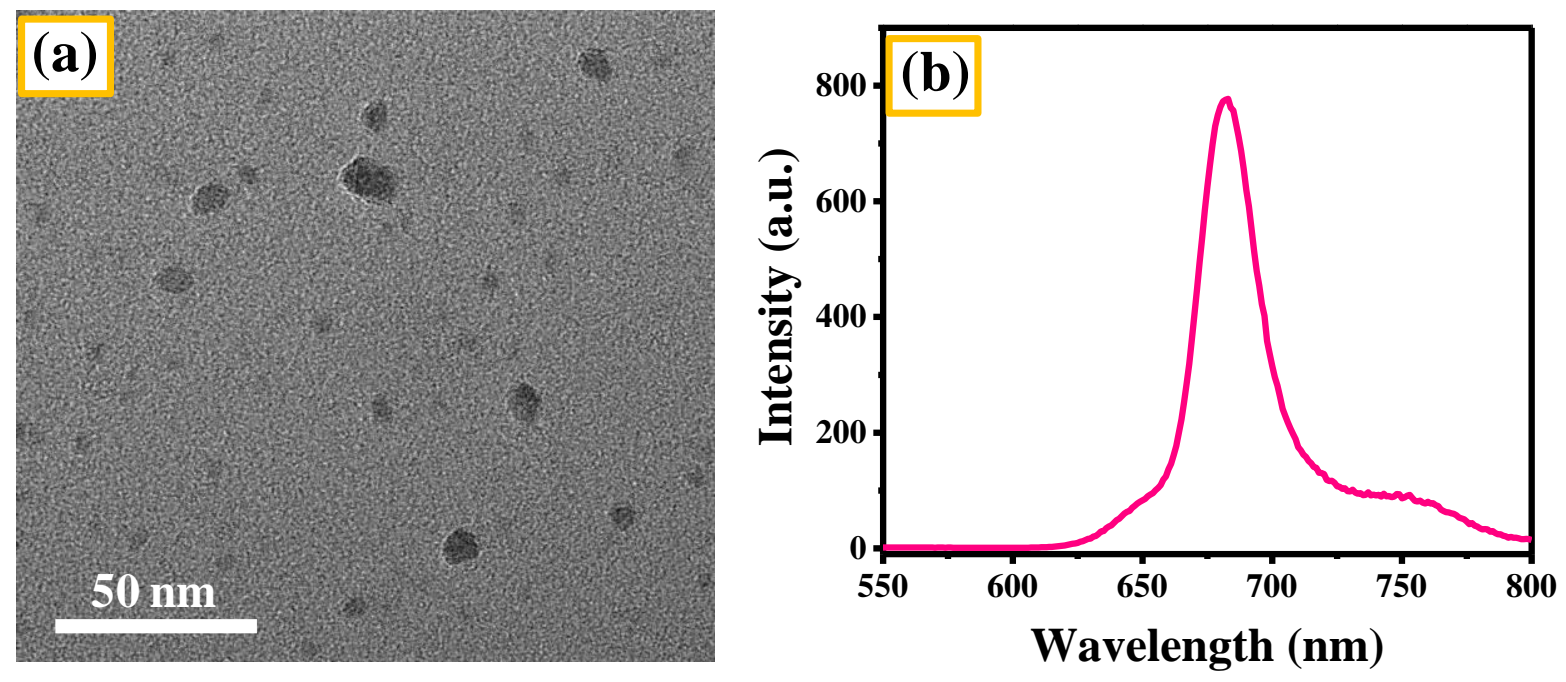

Figure S3. a) TEM image of H-CDs. b) Fluorescence emission spectrum of H-CDs.

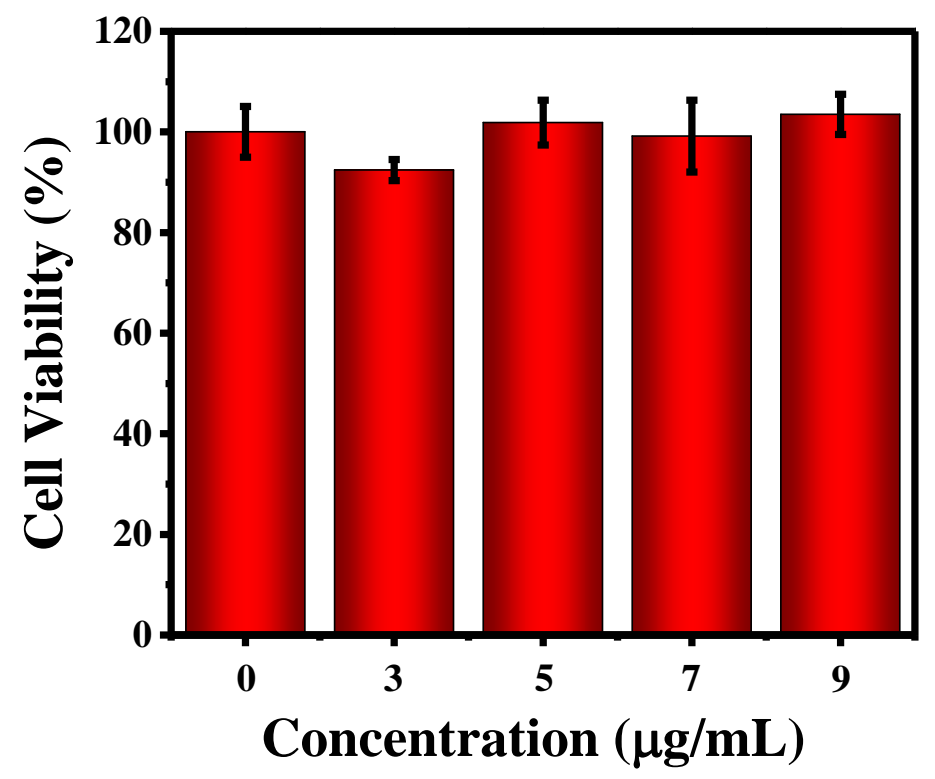

Figure S4. The viability of HeLa cells after incubating with various concentrations of the FR-CDs $(0,3$, 5,7 , and $9 \mu \mathrm{g} / \mathrm{mL}$ ) for $24 \mathrm{~h}$. 


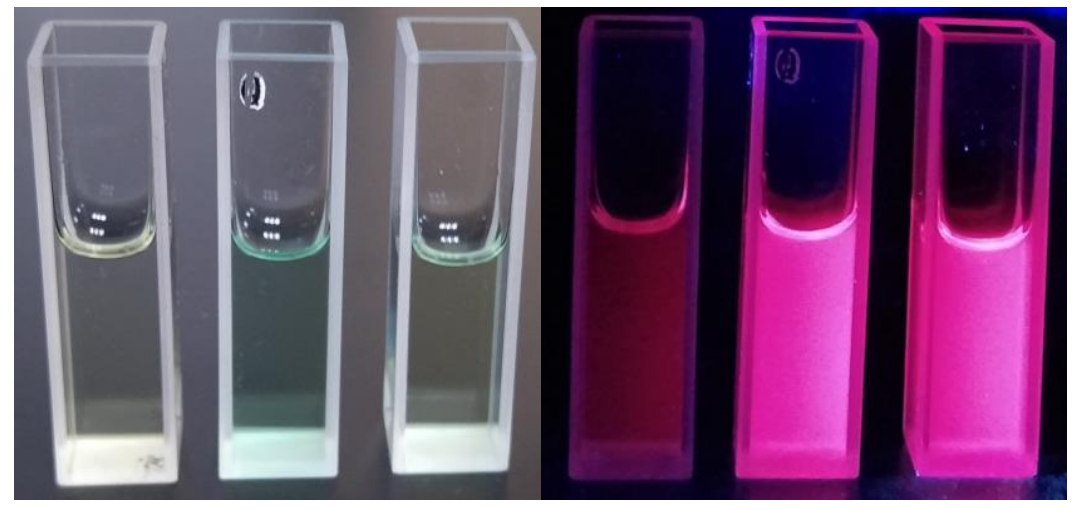

Figure S5. The fluorescence of chloroplasts, FR-CDs and FR-CDs/chloroplast complexes, respectively.
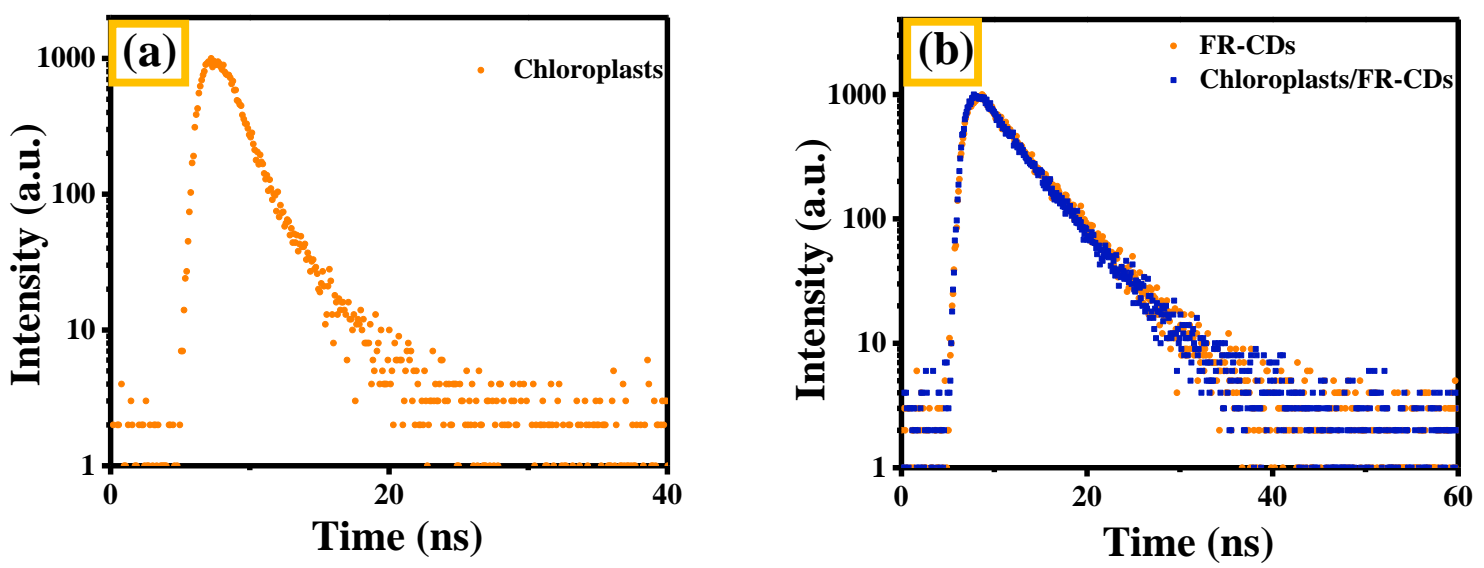

Figure S6. a) Photoluminescence decay curve of chloroplasts monitored at $690 \mathrm{~nm}$. b) PL decay curves of FR-CDs and FR-CDs /chloroplast complexes, monitored at $635 \mathrm{~nm}$. 


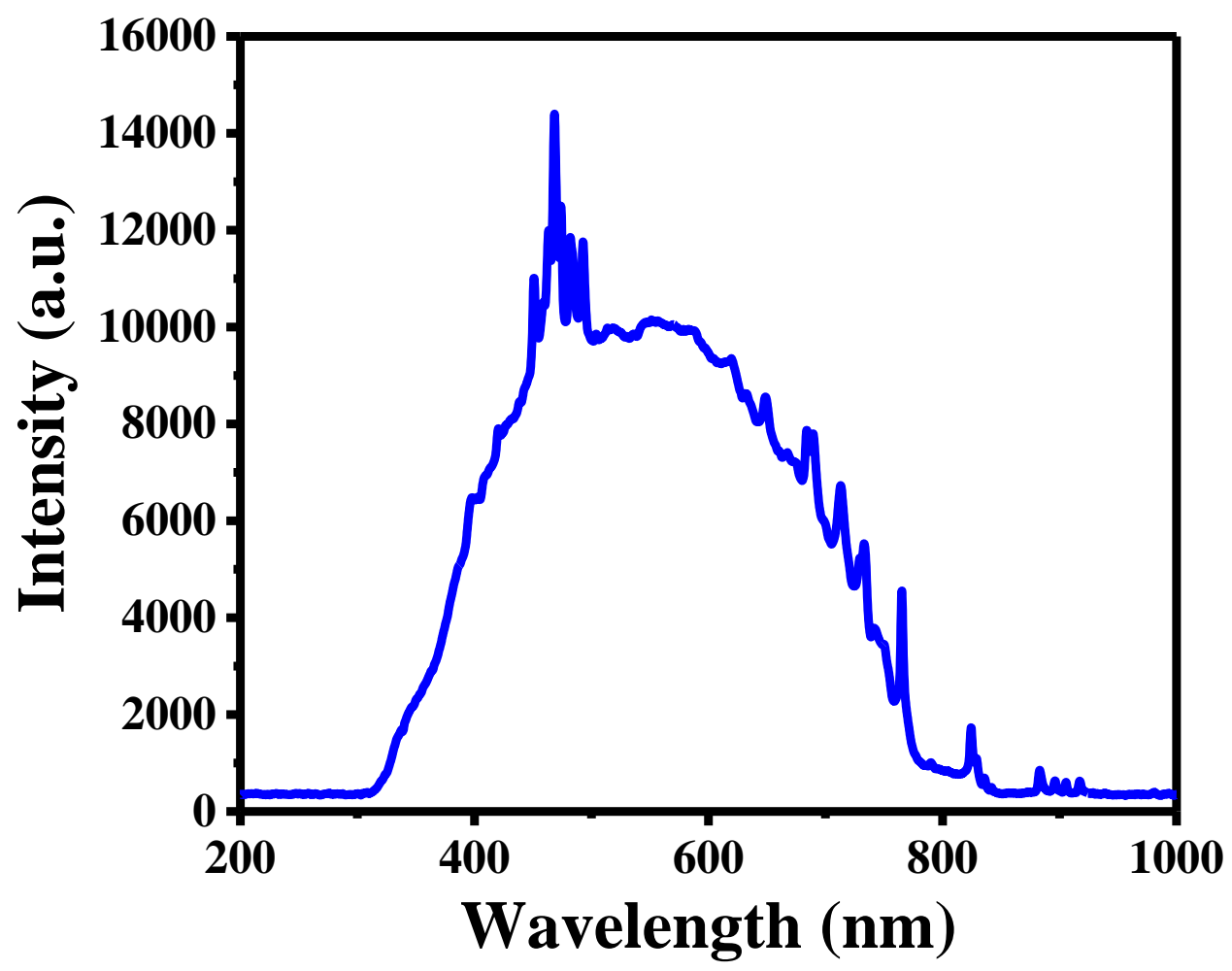

Figure S7. The spectrum of the xenon lamp.
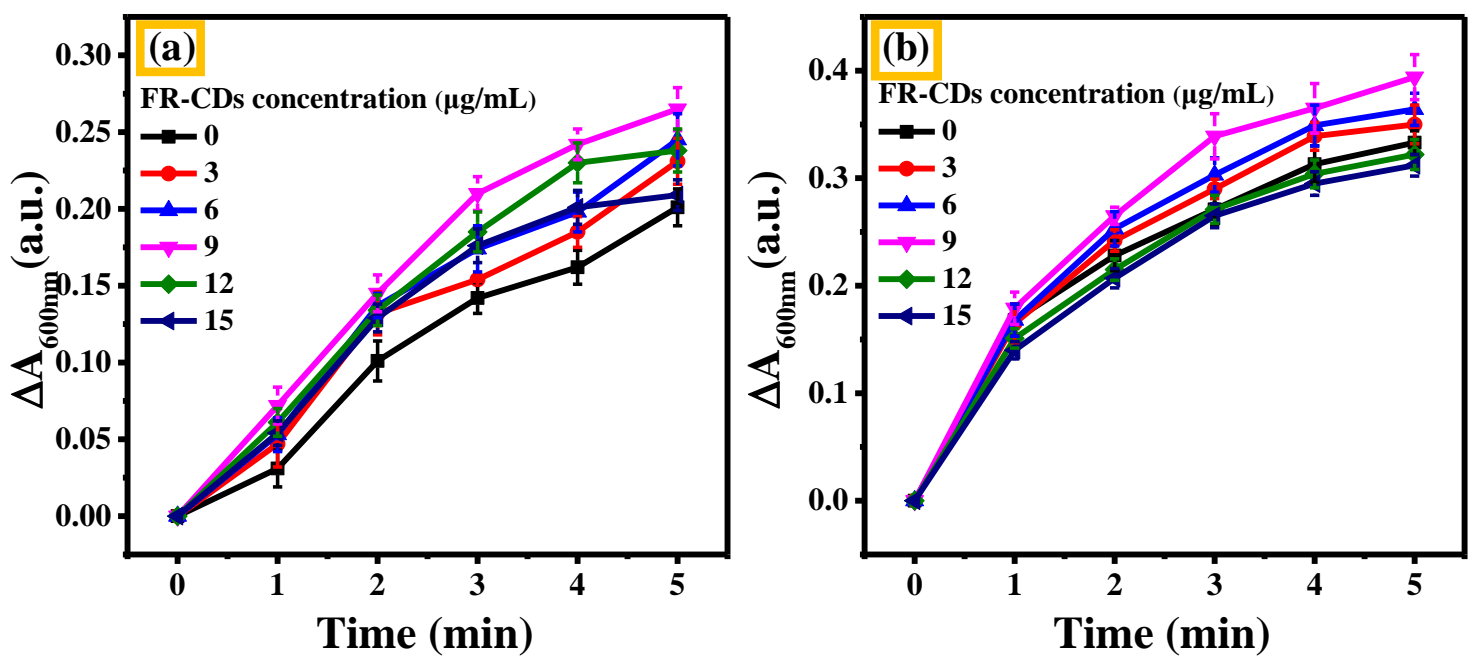

Figure S8. DCPIP reductions under variable concentrations of FR-CDs $(0,3,6,9,12$ and $15 \mu \mathrm{g} / \mathrm{mL})$ in FR-CDs/chloroplast complexes under different light intensity: a) $2 \mathrm{~mW} \mathrm{~cm}^{-2}$, b) $8 \mathrm{~mW} \mathrm{~cm}^{-2}$ 


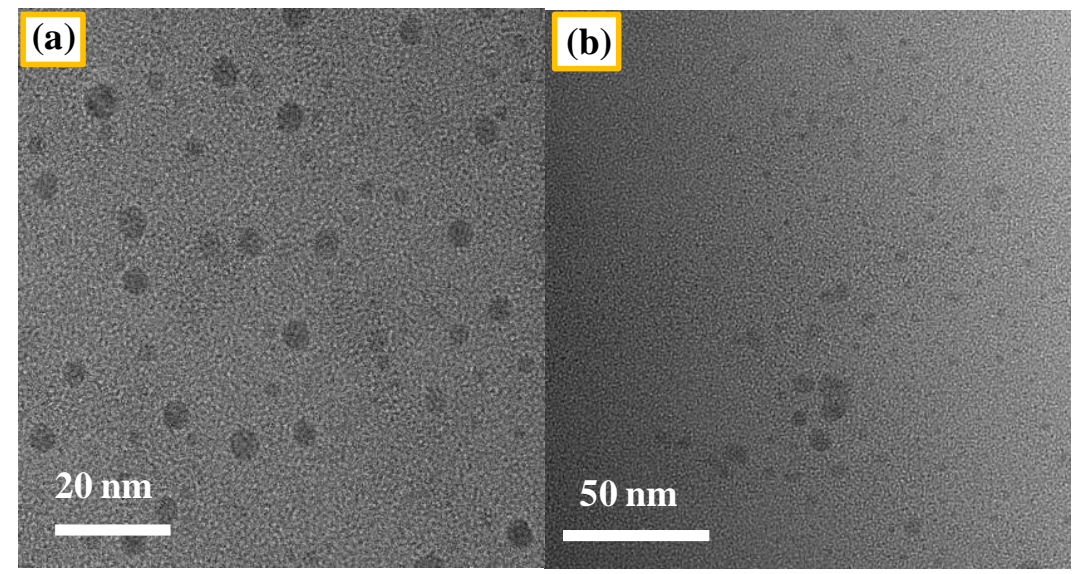

Figure S9. TEM images of B-CDs (a) and R-CDs (b).

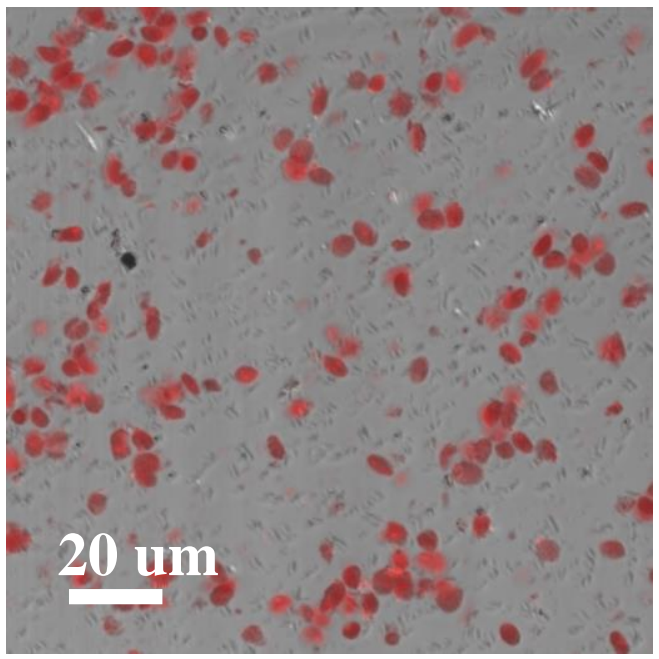

Figure S10. LSM image of the chloroplasts extracted from Roman lettuce.

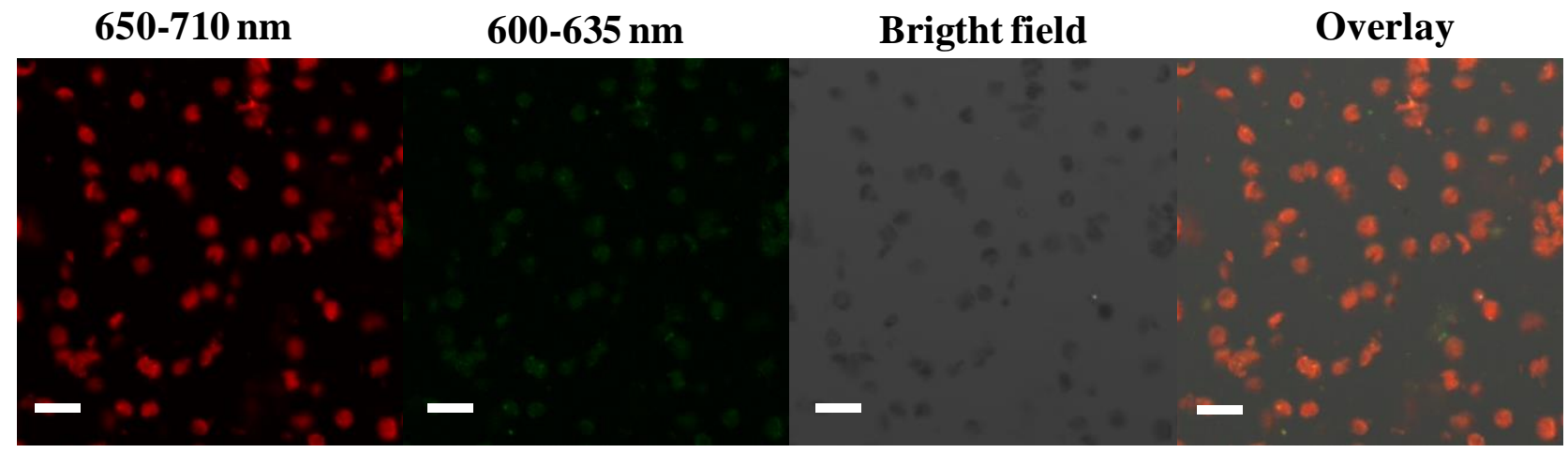

Figure S11. LSM images of FR-CDs/chloroplast complexes collected in the ranges of 650-710 and 
600-635 nm, excited at $405 \mathrm{~nm}$ (scale bar: $10 \mu \mathrm{m}$ ). (In the range of 600-635 nm, the fluorescence of FR-CDs can be collected while the chloroplasts' can't.)

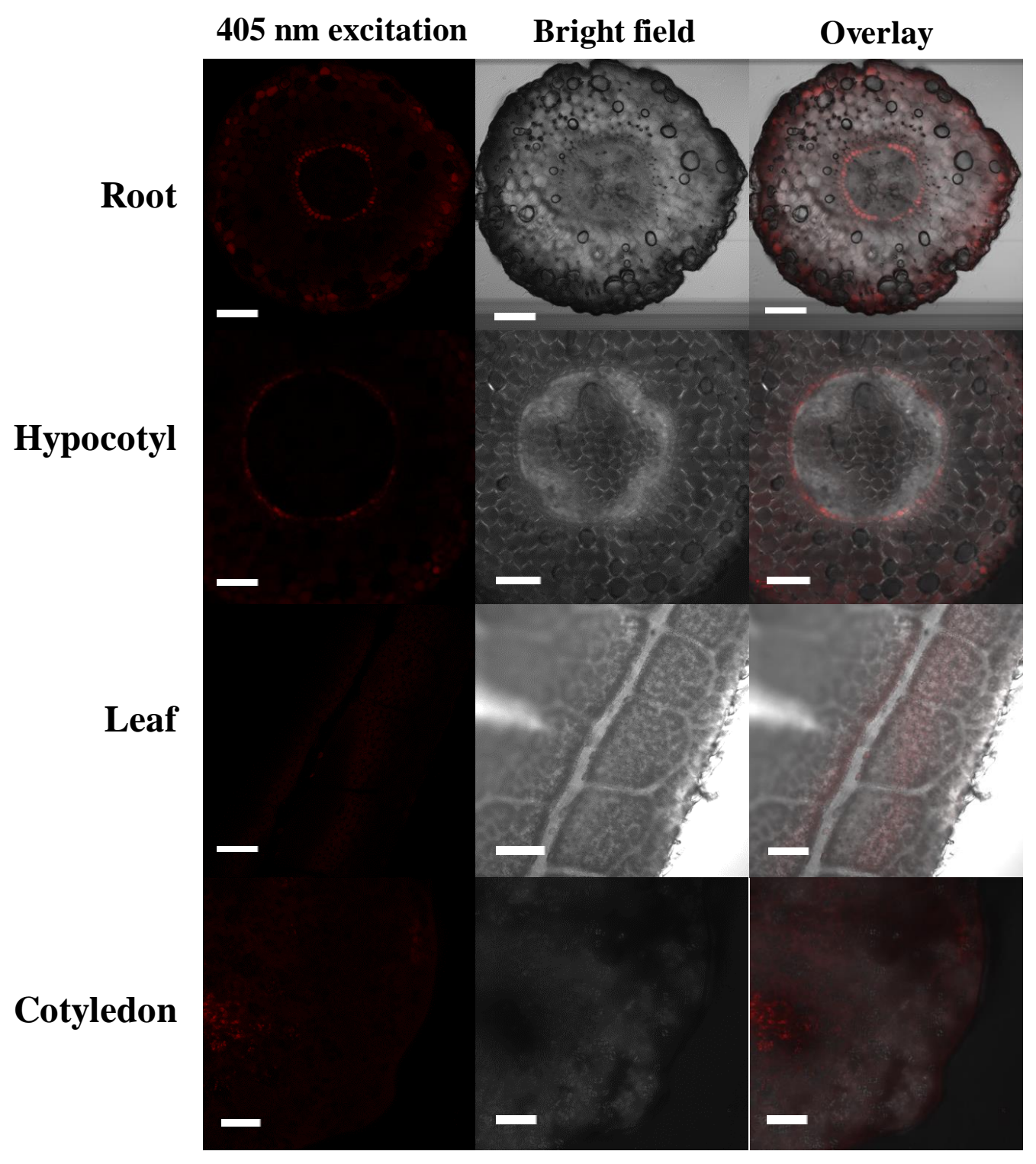

Figure S12. Confocal images of root, stem and leaf of FR-CDs treated (concentration: $10 \mu \mathrm{g} / \mathrm{mL}$ ) lettuce collected in the range of 630-700 nm, excited at $405 \mathrm{~nm}$ (scale bar: $200 \mu \mathrm{m}$ ). 


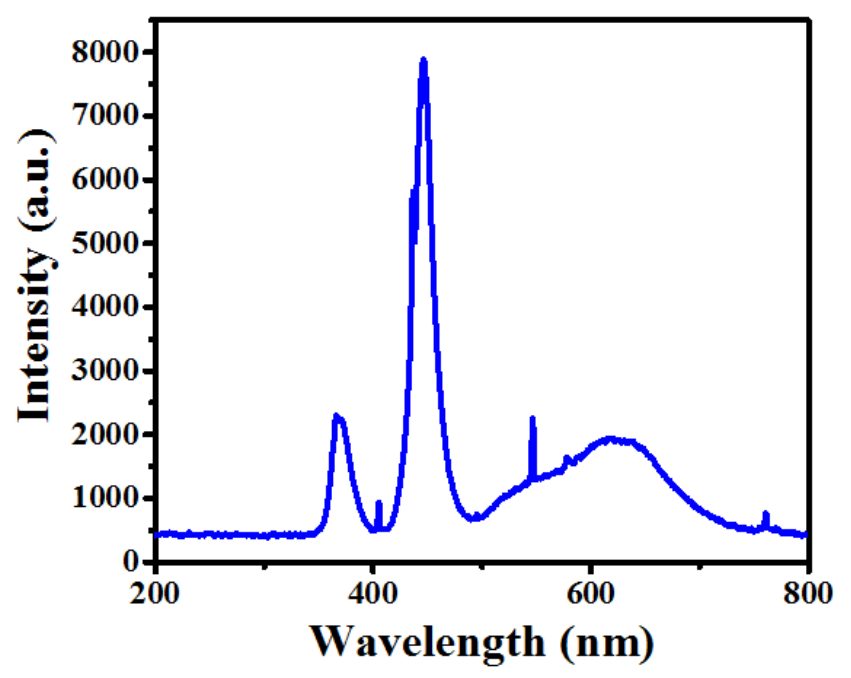

Figure S13. Spectrum of light source (including UV-A light and commercial white light LED) which is used to irradiate the Rome lettuce on the culture shelf. UV-A light: the emission peak at $365 \mathrm{~nm}$; Cmercial white light LED: including blue light and red light.

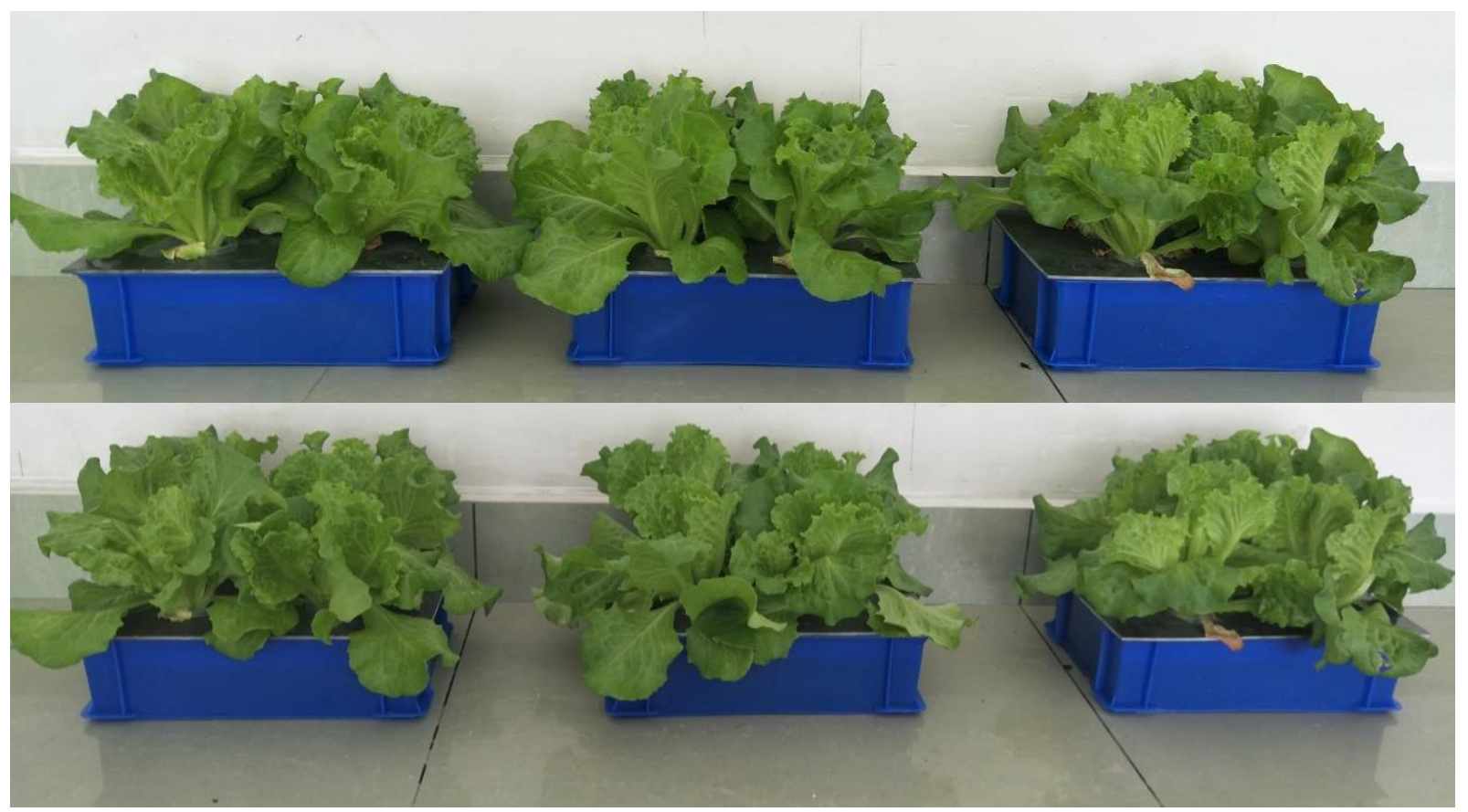

Figure S14. Effect of different concentrations of FR-CDs $(0,4,8 \mu \mathrm{g} / \mathrm{mL}$, from left to right, respectively) on the growth of Rome lettuce under the sunlight. 